\title{
Lichens of the surrounding areas of Termas of Chillán and Las Trancas, Bío-Bío Region, Chile
}

\section{Líquenes de los alrededores de las Termas de Chillán y Las Trancas, Región del Bío-Bío, Chile}

\author{
Iris Pereira ${ }^{1 *}$, Xin Yu Wang ${ }^{2}$, Soon-Ok Oh${ }^{3}$, Pedro Sánchez ${ }^{4}$ \& Jae-Seoun Hur ${ }^{3}$ \\ ${ }^{1}$ Instituto de Ciencias Biológicas, Universidad de Talca, 2 Norte 685, Talca, Chile. \\ ${ }^{2}$ Key Laboratory of Biodiversity and Biogeography, Kunming Institute of Botany, Chinese Academy of Sciences, Yunnan \\ 650204, China. \\ ${ }^{3}$ Korean Lichen Research Institute, Sunchon National University, Sunchon, Korea. \\ ${ }^{4}$ Villa Los Conquistadores, Pasaje 5 1/2 Norte 1991, Talca. \\ *ipereira@utalca.cl
}

\begin{abstract}
The aim of this study was to contribute to the knowledge of the richness and distribution of the lichens in the Bío-Bío Region. A total of 120 samples were collected in the surroundings of Termas de Chillán and Las Trancas, Bio-Bio region. The sampling was realized at random considering the high diversity of available substrates. Taxonomical identification was performed on the basis of the analysis of morphological, reproductive and chromatographic (TLC) characters. A total of 41 species were identified, which include 18 families and 31 genera. Out of the total of identified species, $49 \%$ are corticolous, $34 \%$ saxicolous and $17 \%$ terricolous. Of the total of species found, four are new records for Chile: Menegazzia confusa $\mathrm{P}$. James, M. pertransita (Stirt.) R. Sant., Rinodina sophodes (Ach.) A. Massal. and Usnea sinensis Motyka. For each species, information on lichen name, family, substratum, altitude, global distribution, and sampling site is provided. Also, keys to identify the genera found in the study area are included. Pictures for the new records of lichens for the country are also presented. The work contributes to extend the knowledge on the taxa's distribution in the region. The results suggest that the lichenological expeditions should be continued in unexplored localities of Chile in order to increase the knowledge of the richness, ecology and distribution of lichen biodiversity.
\end{abstract}

KEYwORDs: Chillán, genera key, distribution, ecology, new records.

\section{RESUMEN}

El deseo de este estudio fue contribuir al conocimiento de la riqueza y distribución de los líquenes de la Región del BíoBío. Como producto de una excursión realizada por la mayoría de los autores de este trabajo, un total de 120 muestras fueron recolectadas en los alrededores de las Termas de Chillán y Las Trancas, Región del Bío-Bío, Chile. El muestreo fue realizado al azar considerando la mayoría de los sustratos disponibles. La identificación taxonómica fue establecida en base al análisis de caracteres morfológicos, reproductivos y cromatográficos (CCF). Un total de 41 especies fueron identificadas, las cuales se incluyen en 18 familias y 31 géneros. Del total de las especies identificadas, $49 \%$ son corticícolas, $34 \%$ saxícolas y $17 \%$ terrícolas. Del total de especies encontradas, 4 corresponden a nuevos registros para el país: Menegazzia confusa P. James, M. pertransita (Stirt.) R. Sant., Rinodina sophodes (Ach.) A. Massal. y Usnea sinensis Motyka. Para cada especie se proporciona información acerca del nombre de la especie, familia, sustrato, altitud, distribución global y localidades muestreadas. Se presentan fotografías de los nuevos registros de líquenes para el país. También, se incluyen claves para identificar los géneros encontrados en el área de estudio. El trabajo contribuyó a ampliar el conocimiento acerca de la distribución de los taxones en la región. Los resultados sugieren que expediciones liquenológicas deberían continuar en localidades no exploradas de Chile para incrementar el conocimiento de la riqueza, ecología y distribución de la biodiversidad de líquenes.

Palabras clave: Chillán, clave de géneros, distribución, ecología, nuevos registros. 


\section{INTRODUCTION}

The mainland territory of Chile is divided into 15 regions. Within these regions, there is no clear knowledge of the richness, distribution, and ecology of lichens. Documented literature on these topics is widely dispersed and for this reason it has not been possible to establish a lichen flora at a regional level. The regions where lichen flora have been more intensively studied and reported so far are: Araucania Region (Rubio et al. 2013), Los Lagos Region (Galloway 1992a, Pereira 2007), Aisén Region (Quilhot et al. 2012, Vargas Castillo \& Morano Büchner 2014, Villagra et al. 2009), and Maule Region (Pereira et al. 1999, Pereira \& San Martín 1998). Furthermore, the researchers dedicated to taxonomic studies are very scarce in the country, and the papers available not always give data on the ecology and distribution of the lichen species. In the first contribution about of the lichen flora, ecology and distribution reported for the Bío-Bío Region, only four species of the genus Pseudocyphellaria were cited for the continental territory (Galloway 1992b): P. coriifolia (Müll.Arg.) Malme, P. neglecta (Müll.Arg.) H.Magn., P. nudata (Zahlbr.) D. Galloway and $P$ pilosella Malme. After that, species of genera Cladia, Cladina and Cladonia the genera (Stenroos 1995) has been cited: Cladia aggregata (Sw.) Nyl., Cladina mitis (Sandst.) Hustich. and C. pynoclada (Pers.) Leighton, Cladonia corniculata Ahti et Kashiw., C. didyma (Fée) Vainio, C. gracilis (L.) Willd. subsp. gracilis, C. gracilis (L.) Willd. subsp. valdivensis, C. humilis (With.) J.R.Laundon, C. lepidophora Ahti et Kashiw., C. ochrochlora Flörke, C. phyllophora Hoffm., C. sarmentosa (Hook. f. et Taylor) C.W. Dodge, C. scabriuscula (Delise) Nyl., C. squamosa Hoffm. and C. subchordalis A. Evans. Finally Pereira et al. 2002 have cited epiphytic lichens on Gomortega keule (Molina) Baillon localized into of the Bío-Bío Region as Graphis scripta (L.) Ach., Megalaria grossa (Pers.) Hafellner, Mycomicrothelia thelena (Ach.) D. Hawksw., Parmotrema perlatum (=Parmotrema chinense (Osbeck) Hale \& Ahti), Pertusaria leioplaca DC., Pseudocyphellaria coriifolia (Müll, Arg.) and Thelotrema lepadinum (Ach.) Ach.

The aim of this study is to contribute to the knowledge of the flora, ecology and distribution of the lichens in the BioBio Region. The idea of including genera keys in this study was to fill the gap in lichenological taxonomic literature in the country since the majority of the works lack these tools to carry out taxonomic identification at a genera level. As a way of complementing this gap, the authors of this study proposed the following objectives: a) to identify the lichen species in the studied area, b) to give information about the distribution, ecology and global distribution of the species in the studied area, c) to generate keys to identify the genera found in this study and illustrate new records about lichens for the country.

\section{MATERIALS AND METHODS}

STUDY AREA

The studied area corresponds to the surroundings of the volcanic complex Nevados de Chillán situated at $36^{\circ} 50^{\prime} \mathrm{S}, 7^{\circ} 25^{\prime} \mathrm{W}$, reaching a maximum altitude of $3,212 \mathrm{~m}$. It is located in the transition zone in central Chile with Mediterranean climate vegetation, constituted mainly of sclerophyllous elements and the temperate evergreen forests of southern Chile (Arroyo et al. 2004) (Fig. 1). The combination of biogeographic situation and geomorphologic complexity leads to an exceptional degree of botanical biodiversity including lichens. The forest in this region is dominated by different species of Nothofagus, like Nothofagus obliqua (Mirb.) Oerst., N. pumilio (Poepp et Endl.) Krasser, $N$. antarctica (G. Forst.) Oerst. and $N$. dombeyi (Mirb.) Oerst. with Andean low scrub of Adesmia emarginata Clos, Pozoa coriacea (Lag.) Kuntze, Berberis empetrifolia Lam., Loasa lateritia Lindl. and Chusquea culeou E. Desv.

\section{SAMPLING METHOD}

The sampling was made at random considering the great diversity of available substrates (soil, rocks, bark of trees, and shrubs). The lichens were obtained manually or with chisel and hammer. Each site was georeferenciated with a GPS Model: eTrex Vista ${ }^{\circledR}$ HCx company: Garmin. Data of the habitat, altitude and site names were recorded.

The sampled sites correspond to:

1: Surroundings of Termas de Chillán in three sites,

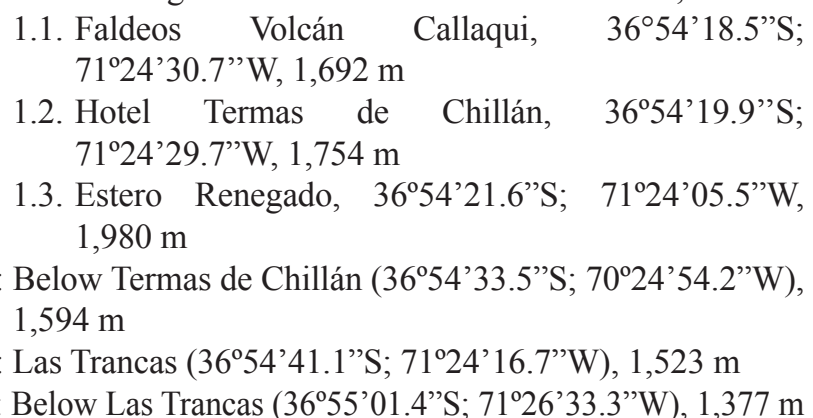

TAXONOMIC IDENTIFICATION

For the taxonomical identification, morphological (vegetative and reproductive) and chromatographic (TLC) (Orange et al. 2010) characters were analysed. The vegetative characters as colour thallus and types of reproductive structures of sexual and asexual origin as perithecia, lirelles or apothecia (lecarorine, biatorin and lecideine), presence or absence of soredia, isidia and picnidia with a binocular stereoscope Kyoma were analysed. The colour, dimensions and form of spores and picnidiospores with a binocular microscope Nikon model Optiphot equipped with a lucida microphotographic camera with graduated ocular were 
studied. Spot tests on different parts of the thallus as upper cortex, medulla and in some structures of the reproductive apparatus as epithecium or amphithecium or hypothecium were made considering the following solutions $\mathrm{K}$ (saturated solution of $\mathrm{KOH}$ to $20 \%$ ), $\mathrm{C}$ (aqueous solution of sodium hypochlorite 50\%), KC (application solution $\mathrm{K}$ following C), $\mathrm{P}$ (alcohol solution of paraphenylendiamine) and aqueous solution of concentrated nitric acid to $60 \%$. The lichen substances were determined by thin layer chromatography (TLC) using a glass plate coated with TLC Silica gel 60 in solvent systems A (toluene:dioxin:acetic = 180:45:5 (Orange et al. 2010). The studied material was deposited in the Herbarium of the Korea Lichen Research Institute (KoLRI) Suncheon National University (Korea) and duplicates were deposited at the Herbarium of the Talca University Talca Chile).

\section{RESULTS AND DISCUSSION}

LICHEN RICHNESS

A total of 41 species, belonging to 18 families and 31 genera were identified, of which $49 \%$ are corticolous, $34 \%$ saxicolous, and 17 terricolous (Fig. 2). The studied species are listed in Table I.

The Parmeliaceae family was represented by 12 species, followed by Lecanoraceae ( 5 species), Lobariaceae and Peltigeraceae (4 species); Stereocaulaceae and Umbilicariaceae ( 2 species), and the rest families by only 1 species. The species richness of each family is represented in Figure 3.

Four new records are mentioned for the country: Menegazzia confusa P. James (Fig. 4, A), M. pertransita (Stirt.) R. Sant. (Fig. 4, B), Rinodina sophodes (Ach.) A. Massal. (Fig. 4, C), and Usnea sinensis Motyka (Fig. 4, D).

\section{DisTRIBUTION OF THE SPECIES}

Of the four new records of lichens found in the studied area, the Menegazzia species appear as epiphytes on Nothofagus antarctica between 1,337 to $1,523 \mathrm{~m}$. Menegazzia confusa is currently known only from Australia (Kantvilas \& James 1987), therefore these results extend its global distribution. On the other hand, M. pertransita is known from Australia, New Zealand, South America, and Antarctica (Santesson 1942). Nevertheless, it has been difficult to pinpoint with exactitude the distribution of this species in South America until now, due to the lack of information about where it was found. Rinodina sophodes is known in the Northern Hemisphere and cited in Italy on Quercus pubescens Willd. (Rizzi et al. 2011) and in France (Roux 2012). Usnea sinensis has been cited on wood in China (Zahlbruckner 1938-1940) and in India (Western Ghats and Karnataka)
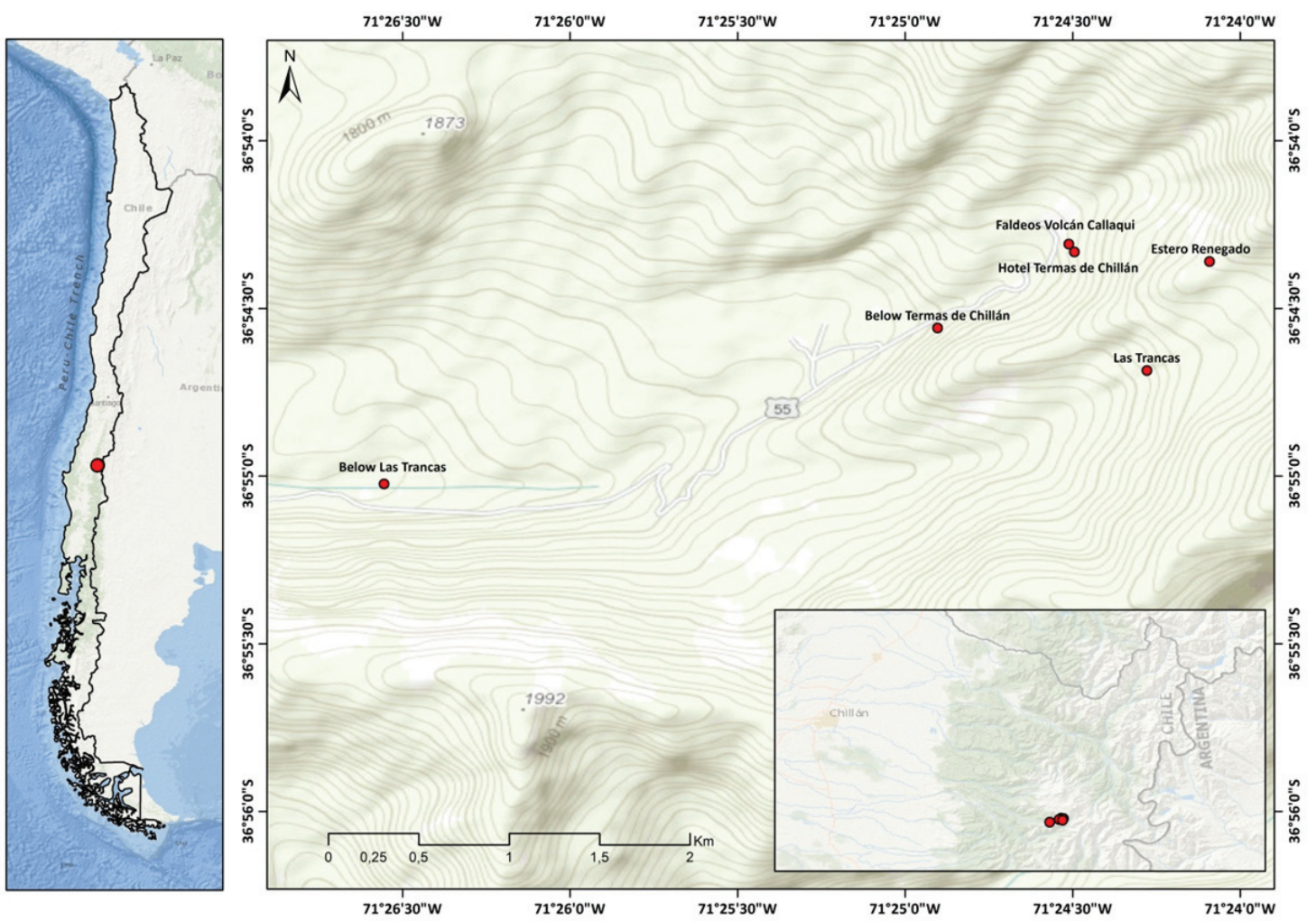

Figure 1. Studied area. The number show the collection sites.

Figura 1. Área de estudio. Los números muestran los sitios de recolección. 
growing on toddy palm bark (Vinayaka et al. 2012).

The following species extend its northern distribution in Chile: Bryoria chalybeiformis (L.) Brodo \& D. Hawksw., Cetraria aculeata (Schreb.) Fr., Lecanora argentata (Ach.) Malme, Leptogium cochleatum (Dicks.) P.M. Jørg. \& P. James, Peltigera collina (Ach.) Schrad., P. neckeri (Ach.) Schrad. and Umbilicaria nylanderiana (Zahlbr.) H. Magn.

Pseudocyphellaria dissimilis (Nyl.) D.J. Galloway \& P. James has only been cited in Chile in Aisén Region and Crocodia guilleminii (Mont.) Nyl. is distributed from Conguillio National Park to Magallanes (Galloway \& Elix, 2013, Quilhot et al. 2012), therefore the expansion of their distribution is only continental since both species also occur on Juan Fernández Islands (Galloway 1992b).

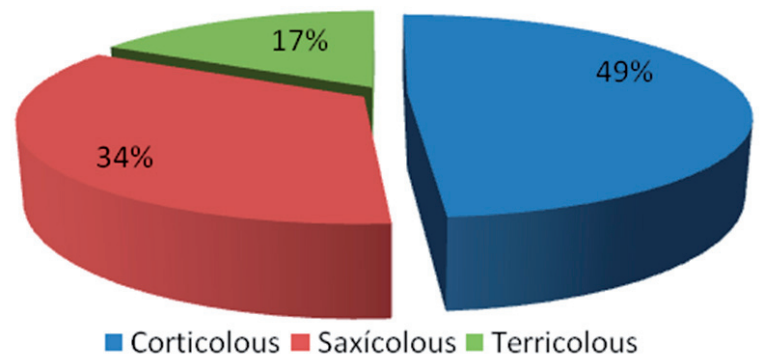

FIGURE 2. Distribution of lichen species by substrate in studied area.

FiguRA 2. Distribución de las especies de líquenes por substrato en el área de estudio.

TABLE I. Lichen species from the surrounding areas of Termas de Chillán and Las Trancas (Bío-Bío Region, Chile). Data referring the substrate, altitude, and collection site where they were found and its global distribution are detailed.

Tabla I. Especies de líquenes de los alrededores de Termas de Chillán y Las Trancas (Región del Bío-Bío, Chile). Se detallan datos referentes al substrato, altitud y sitio de recolección donde fueron encontradas y su distribución global.

\begin{tabular}{|c|c|c|c|c|c|c|}
\hline & Lichen Species & FAMILY & Substrates & $\begin{array}{l}\text { Altitude } \\
\quad(\mathrm{M})\end{array}$ & Global Distribution & $\begin{array}{l}\text { COLLECTION } \\
\quad \text { SITE }\end{array}$ \\
\hline 1 & $\begin{array}{l}\text { Acarospora schleicheri (Ach.) A. } \\
\text { Massal. }\end{array}$ & Acarosporaceae & On rocks & 1980 & $\begin{array}{l}\text { Europe, Africa, North } \\
\text { and South America }\end{array}$ & 1.1 \\
\hline 2 & $\begin{array}{l}\text { Blastenia ferruginea (Huds.) A. } \\
\text { Massal }\end{array}$ & Teloschistaceae & Trunk & 1692 & Cosmopolitan & 1.3 \\
\hline 3 & $\begin{array}{l}\text { Bryoria chalybeiformis (L.) Brodo } \\
\text { \& D. Hawksw. ** }\end{array}$ & Parmeliaceae & Trunk & 1692 & Bipolar & 1.3 \\
\hline 4 & Buellia cf. hyperbolica Bagl. & Caliciaceae & Trunk & 1523 & $\begin{array}{l}\text { United Kingdom and } \\
\text { Chile }\end{array}$ & 3 \\
\hline 5 & $\begin{array}{l}\text { Catapyrenium cinereum (Pers.) } \\
\text { Körb. }\end{array}$ & Verrucariaceae & Soil & 1594 & $\begin{array}{l}\text { Native of North } \\
\text { America }\end{array}$ & 2 \\
\hline 6 & Cetraria aculeata (Schreb.) Fr. ** & Parmeliaceae & Trunk & $\begin{array}{l}1980,1754 \\
1692\end{array}$ & Bipolar & $1.1,1.2,1.3$ \\
\hline 7 & $\begin{array}{l}\text { Circinaria calcarea (L.) A. Nordin, } \\
\text { S. Savić \& Tibell }\end{array}$ & Megasporaceae & On rocks & 1980 & Cosmopolitan & 1.1 \\
\hline 8 & $\begin{array}{l}\text { Crocodia guilleminii (Mont.) } \\
\text { Nyl.,** }\end{array}$ & Lobariaceae & Trunk & 1337 & $\begin{array}{l}\text { Endemic to southern } \\
\text { South America }\end{array}$ & 4 \\
\hline 9 & Hypotrachyna sinuosa $(\mathrm{Sm}$.) Hale & Parmeliaceae & Trunk & 1523 & Cosmopolitan & 3 \\
\hline 10 & $\begin{array}{l}\text { Ingvariella bispora (Bagl.) } \\
\text { Guderley \& Lumbsch }\end{array}$ & Thelotremaceae & On rocks & 1584 & Subcosmopolitan & 2 \\
\hline 11 & $\begin{array}{l}\text { Lecanora argentata (Ach.) } \\
\text { Degel.** }\end{array}$ & Lecanoraceae & Trunk & 1692 & Cosmopolitan & 1.3 \\
\hline 12 & Lecanora chlarotera Nyl. & Lecanoraceae & Trunk & 1594 & Subcosmopolitan & 2 \\
\hline 13 & Lecidea capensis Zahlbr. & Lecideaceae & On rock & 1980 & $\begin{array}{l}\text { Australia, New } \\
\text { Zealand, South Africa } \\
\text { and Chile }\end{array}$ & 1.1 \\
\hline
\end{tabular}




\begin{tabular}{|c|c|c|c|c|c|c|}
\hline & LiCHEN SPECIES & FAMILY & Substrates & $\begin{array}{l}\text { Altitude } \\
\text { (M) }\end{array}$ & Global Distribution & $\begin{array}{l}\text { Collection } \\
\text { Site }\end{array}$ \\
\hline 14 & $\begin{array}{l}\text { Lecidella elaeochroma (Ach.) M. } \\
\text { Choisy }\end{array}$ & Lecanoraceae & Trunk & $\begin{array}{l}1754,1692 \\
1523,1337\end{array}$ & Subcosmopolitan & $1.2,1.3,3,4$ \\
\hline 15 & Lepraria lobificans Nyl. & Stereocaulaceae & Soil & 1594 & Cosmopolitan & 2 \\
\hline 16 & $\begin{array}{l}\text { Leptogium cochleatum (Dicks.) } \\
\text { P.M. Jørg. ** }\end{array}$ & Collemataceae & Trunk & 1523,1337 & $\begin{array}{l}\text { Tropical and temperate } \\
\text { regions }\end{array}$ & 3,4 \\
\hline 17 & $\begin{array}{l}\text { Melanelia cf. exasperatula (Nyl.) } \\
\text { Essl. }\end{array}$ & Parmeliaceae & Trunk & 1523 & $\begin{array}{l}\text { Northern Hemisphere } \\
\text { and Chile }\end{array}$ & 3 \\
\hline 18 & Menegazzia confusa P. James* & Parmeliaceae & Trunk & 1337,1523 & Australia & 3,4 \\
\hline 19 & $\begin{array}{l}\text { Menegazzia pertransita (Stirt.) R. } \\
\text { Sant. * }\end{array}$ & Parmeliaceae & Trunk & 1523 & $\begin{array}{l}\text { New Zealand, } \\
\text { Australia, and South } \\
\text { America }\end{array}$ & 3 \\
\hline 20 & $\begin{array}{l}\text { Montanelia panniformis Nyl.) } \\
\text { Divakar, A. Crespo, Wedin \& Essl. }\end{array}$ & Parmeliaceae & On rock & 1980 & $\begin{array}{l}\text { North America and } \\
\text { Eurasia }\end{array}$ & 1.1 \\
\hline 21 & $\begin{array}{l}\text { Neuropogon antarcticus (Du Rietz) } \\
\text { I.M. Lamb }\end{array}$ & Parmeliaceae & On rock & 1980 & $\begin{array}{l}\text { Subantartic and } \\
\text { circumpolar }\end{array}$ & 1.1 \\
\hline 22 & Ochrolechia parella (L.) A. Massal. & Ochrolechiaceae & On rock & 1980 & Bipolar & 1.1 \\
\hline 23 & Parmelia sulcata Taylor & Parmeliaceae & Trunk & 1523 & Cosmopolitan & 3 \\
\hline 24 & $\begin{array}{l}\text { Peltigera cf. austroamericana } \\
\text { Zahlbr. }\end{array}$ & Peltigeraceae & Soil & 1692 & $\begin{array}{l}\text { Central and South } \\
\text { America }\end{array}$ & 1.3 \\
\hline 25 & Peltigera collina (Ach.) Schrad. ** & Peltigeraceae & Soil & 1692,1337 & Circumpolar & $1.3,4$ \\
\hline 26 & $\begin{array}{l}\text { Peltigera didactyla (With.) J.R. } \\
\text { Laundon }\end{array}$ & Peltigeraceae & Soil & 1594 & Cosmopolitan & 2 \\
\hline 27 & $\begin{array}{l}\text { Peltigera neckeri Hepp ex Müll. } \\
\text { Arg. ** }\end{array}$ & Peltigeraceae & Soil & 1337 & $\begin{array}{l}\text { Temperate and boreal } \\
\text { regions of North } \\
\text { America, Europe and } \\
\text { Asia }\end{array}$ & 4 \\
\hline 28 & $\begin{array}{l}\text { Pertusaria cf. albescens (Huds.) M. } \\
\text { Choisy \& Werner }\end{array}$ & Pertusariaceae & Trunk & 1523 & Bipolar & 3 \\
\hline 29 & $\begin{array}{l}\text { Protousnea poeppigii (Nees \& } \\
\text { Flot.) Krog }\end{array}$ & Parmeliaceae & Trunk & 1692 & $\begin{array}{l}\text { Endemic to southern } \\
\text { South America }\end{array}$ & 1.3 \\
\hline 30 & $\begin{array}{l}\text { Pseudocyphellaria dissimilis (Nyl.) } \\
\text { D.J. Galloway ** }\end{array}$ & Lobariaceae & Trunk & 1337 & Paleotropical & 4 \\
\hline 31 & $\begin{array}{l}\text { Pseudocyphellaria neglecta (Müll. } \\
\text { Arg.) H. Magn. }\end{array}$ & Lobariaceae & Soil & 1337 & Austral & 4 \\
\hline 32 & $\begin{array}{l}\text { Pseudocyphellaria nudata (Zahlbr.) } \\
\text { D. J. Galloway }\end{array}$ & Lobariaceae & Trunk & 1523 & $\begin{array}{l}\text { Endemic to southern } \\
\text { South America }\end{array}$ & 3 \\
\hline 33 & Rhizocarpon geographicum (L.) DC & Rhizocarpaceae & On rock & 1980 & Cosmopolitan & 1.1 \\
\hline
\end{tabular}




\begin{tabular}{|c|c|c|c|c|c|c|}
\hline & Lichen Species & FAMILY & Substrates & $\begin{array}{l}\text { Altitude } \\
\text { (M) }\end{array}$ & Global Distribution & $\begin{array}{l}\text { COLLECTION } \\
\text { SITE }\end{array}$ \\
\hline 34 & $\begin{array}{l}\text { Rhizoplaca melanophthalma } \\
\text { (Ramond) Leuckert \& Poelt }\end{array}$ & Lecanoraceae & On rock & 1980 & $\begin{array}{l}\text { Bipolar, circumboreal, } \\
\text { extending to tropic in } \\
\text { alpine areas }\end{array}$ & 1.1 \\
\hline 35 & $\begin{array}{l}\text { Rinodina sophodes (Ach.) A. } \\
\text { Massal.* }\end{array}$ & Lecanoraceae & Trunk & 1523 & Bipolar & 3 \\
\hline 36 & $\begin{array}{l}\text { Squamarina squamulosa (Nyl.) } \\
\text { Follmann }\end{array}$ & Stereocaulaceae & On rock & 1594 & Bipolar & 2 \\
\hline 37 & $\begin{array}{l}\text { Tephromela atra (Huds.) Hafellner } \\
\text { in K. Kalb. }\end{array}$ & Tephromelataceae & On rock & 1980 & Cosmopolitan & 1.1 \\
\hline 38 & $\begin{array}{l}\text { Umbilicaria krascheninnikovii } \\
\text { (Savicz) Zahlbr. }\end{array}$ & Umbilicariaceae & On rock & 1980 & $\begin{array}{l}\text { North and South } \\
\text { America and Asia }\end{array}$ & 1.1 \\
\hline 39 & $\begin{array}{l}\text { Umbilicaria nylanderiana (Zahlbr.) } \\
\text { H. Magn. } * *\end{array}$ & Umbilicariaceae & On rock & 1980 & Bipolar & 1.1 \\
\hline 40 & Usnea sinensis Motyka* & Parmeliaceae & Trunk & 1692 & China and Chile & 1.3 \\
\hline 41 & $\begin{array}{l}\text { Xanthoparmelia mougeotii } \\
\text { (Schaer.) Hale }\end{array}$ & Parmeliaceae & On rock & 1980 & $\begin{array}{l}\text { Pantemperate to } \\
\text { subarctic, Europe, } \\
\text { Asia, southern Africa } \\
\text { and western North } \\
\text { America and South } \\
\text { America }\end{array}$ & 1.1 \\
\hline
\end{tabular}

*: New record for Chile. / Nuevo registro para Chile

**: Extension of geographic distribution in Chile. / Extensión de la distribución geográfica en Chile.

Collection sites: 1: Surroundings of Termas de Chillán: 1.1, 1.2 and 1.3; 2: Below Termas de Chillán; 3: Las Trancas; 4: Below Las Trancas, Bio-Bio Region. / Sitios de recolección: 1: Alrededores de las Termas de Chillán: 1.1, 1.2 y 1.3; 2: bajo las Termas de Chillán; 3: Las Trancas; 4: bajo Las Trancas, Región del Bío-Bío.

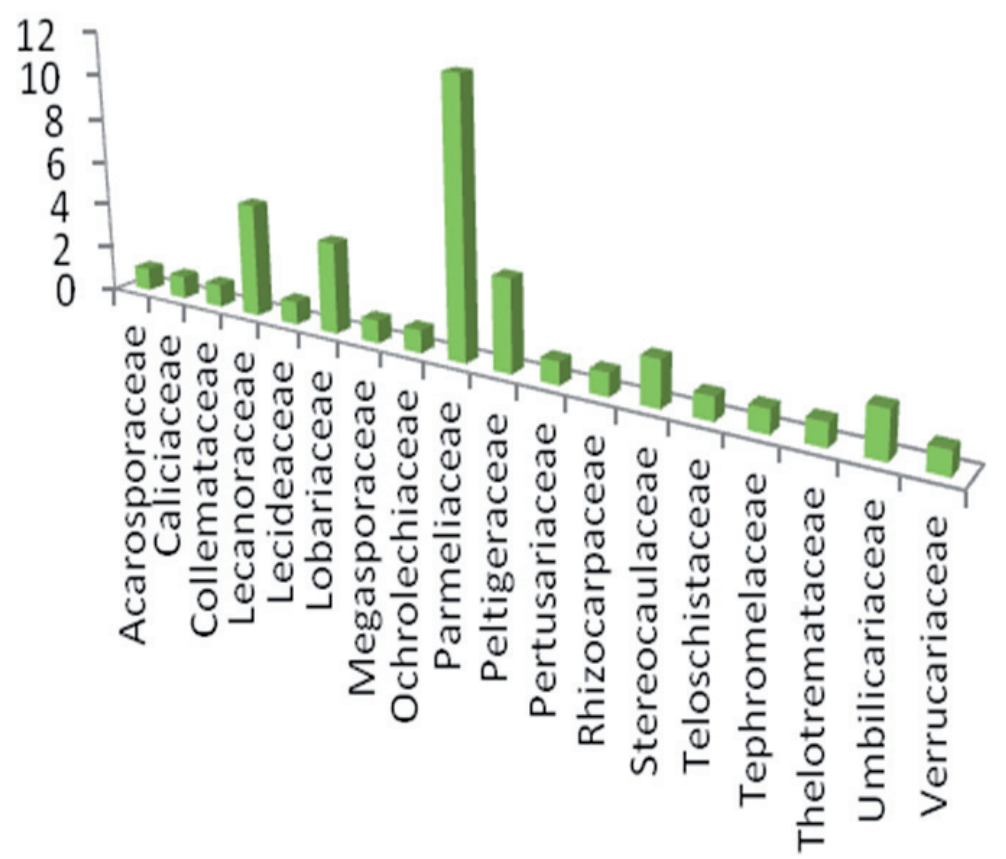

FIGURE 3. Distribution of the lichen richness by family in the studied area.

FIgURA 3. Distribución de la riqueza liquénica por familia en el área de estudio. 


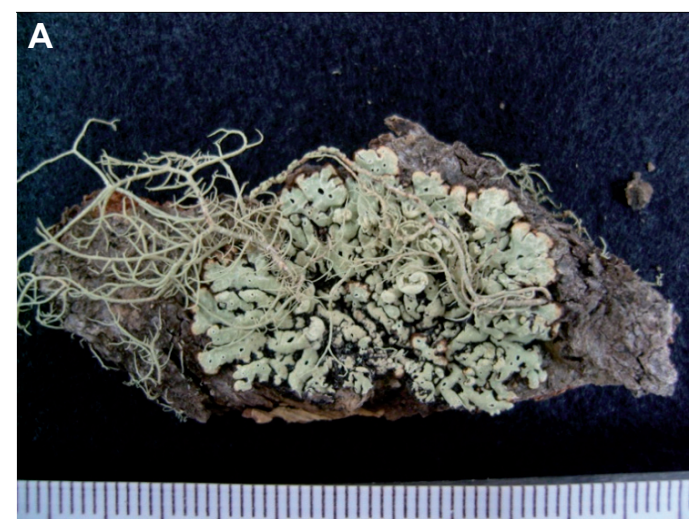

Menegazzia confusa

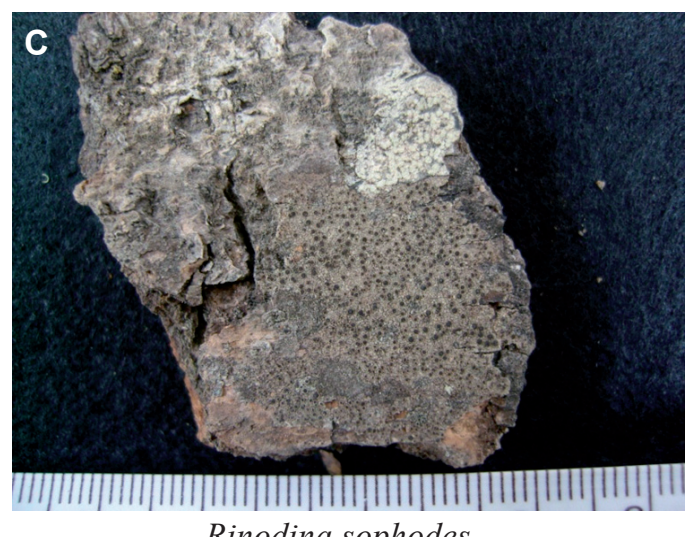

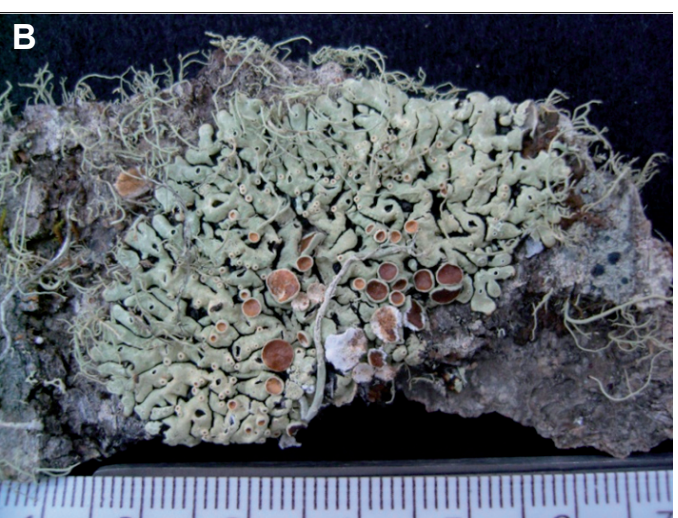

Menegazzia pertransita

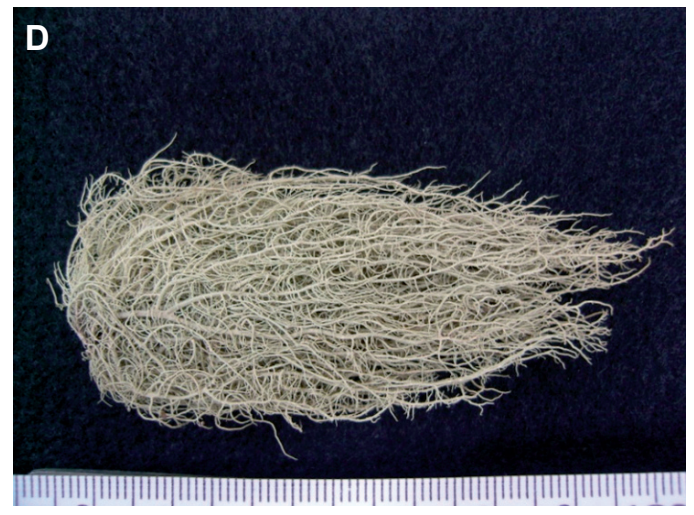

Usnea sinensis

FiguRE 4. New records for Chile: lichen thallus and their substrates. A- Menegazzia confusa; B- Menegazzia pertransita; C- Rinodina sophodes; D- Usnea sinensis.

Figura 4. Nuevos registros para Chile: talos liquénicos y sus substratos. A- Menegazzia confusa; B- Menegazzia pertransita; C- Rinodina sophodes; D- Usnea sinensis.

Tephromela atra (Huds.) Hafellner has been found in Chile on tree bark and shrubs, and rocky substrates (Vargas $\&$ Morano 2014). In the locality finding, this species also appears growing on rocks.

Altitudinal RANGe of THE LiChEN SPECIES

There is a clear relationship between the altitude and the substrates on which grows the lichens. Of this way, the saxicolous and terricolous lichen are concentrated between 1700 to $1960 \mathrm{~m}$ of altitude while corticolous lichen is concentrated between 1300 to $1560 \mathrm{~m}$ corresponding to arboreal and shrubs strata. Protousnea poeppigii (Nees \& Flot.) Krog is the species best represented in the arboreal stratum as well as also species of the Usnea genus, which grow mixed and it could be easily mistaken for a beginner. Acarospora schleicheri (Ach.) A. Massal., Circinaria calcarea (L.) A. Nordin, S. Savić \& Tibell, C. aculeata (Schreb.) Fr., Lecidea capensis Zahlbr., Montanelia panniformis (Nyl.) Divakar, A. Crespo, Wedin \& Essl., Neuropogon antarcticus (Du Rietz) I.M. Lamb, Ochrolechia parella (L.) A. Massal., Rhizocarpon geographicum (L.) DC., Rhizoplaca melanophthalma (DC.) Leuckert \& Poelt, Tephromela atra (Huds.) Hafellner, Umbilicaria krascheninnikovii (Savicz) Zahlbr., U. nylanderiana (Zahlbr.) H. Magn., and Xanthoparmelia mougeotii (Schaer.) Hale were the species found at a higher altitude $(1,980 \mathrm{~m})$, occurring on rocky substrates.

Among the species found at lower altitudes $(1,337$ m), Lecidella elaeochroma (Ach.) Hazsl., Leptogium cochleatum (Dicks.) P.M. Jørg. \& P. James, Menegazzia confusa P. James, Pseudocyphellaria dissimilis (Nyl.) D.J. Galloway \& P. James, C. guilleminii (Mont.) Nyl. and $P$. neglecta (Müll.Arg.) Vain., grows on tree and shrubs barks, while Peltigera collina (Ach.) Schrad, and P. neckeri Hepp. ex Müll.Arg. on sandy soils. 
Lichens in Chillán, Bío-Bío region: PereIRA, I. ET AL.

KEYS TO IDENTIFY OF LICHEN GENERA IN THE STUDIED AREA

The keys presented here are based on morphological and reproductive characters studied. The type of substrate colonized for the lichens were also considered.

LiCHENS WITH CRUSTOSE THALLUS

1. Thallus yellowish, lemon yellow or more rare beige. .2

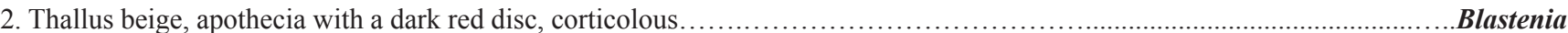

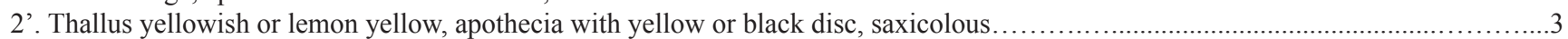

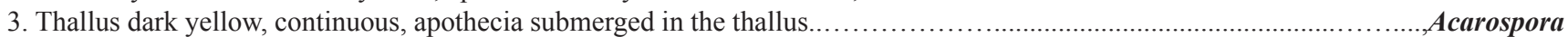

3'. Thallus lemon green, areolate, black lecideine apothecia between the areolae, not submerged in the thallus................Rhizocarpon

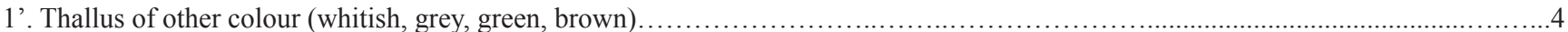

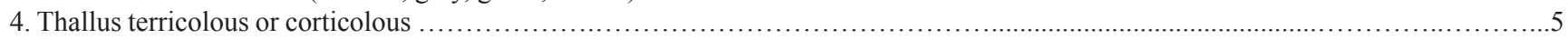

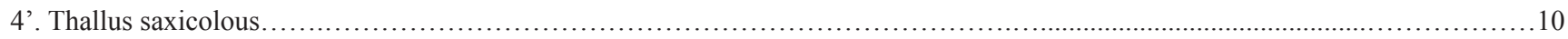

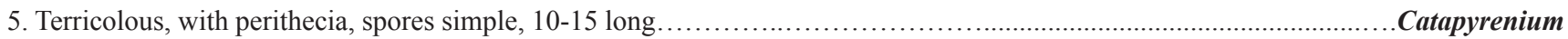

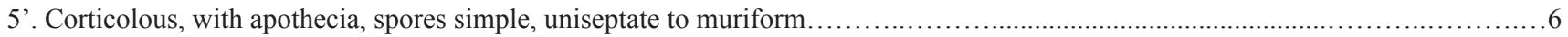

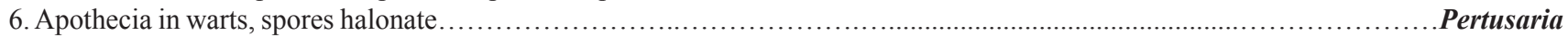

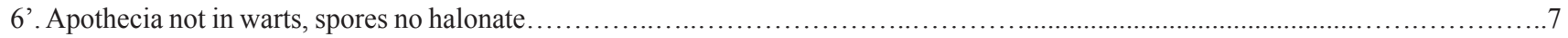

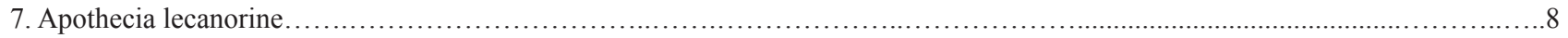

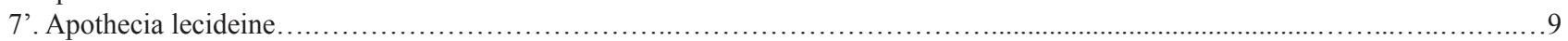

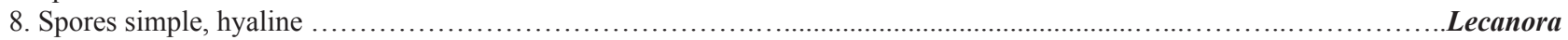

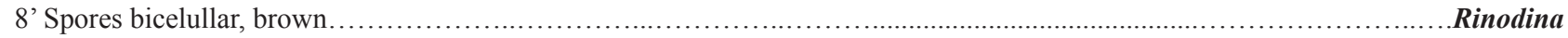

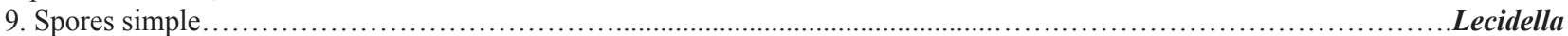

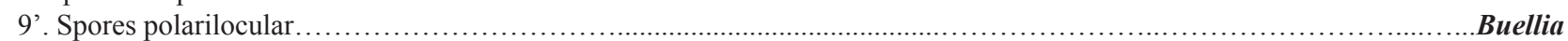

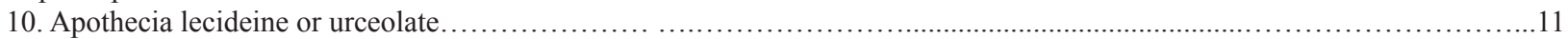

11. Apothecia lecideine, spores simple or uniseptate, hyaline, $5-25 \mu \mathrm{m}$ long ........................................................ecidea

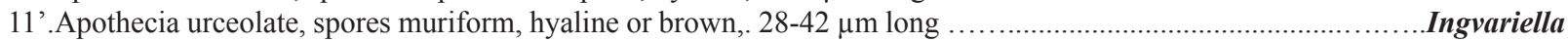

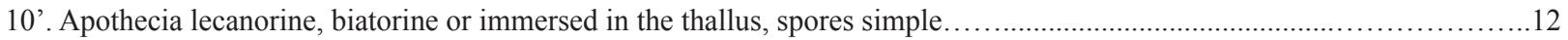

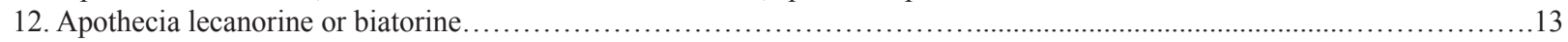

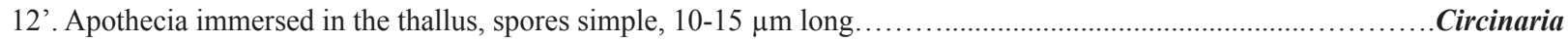

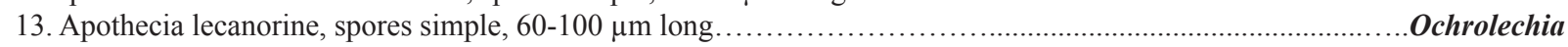

13'. Apothecia lecanorine or biatorine, spores simple, $10-15 \mu \mathrm{m}$ long............................................................Tephromela

LiCHENS WITH FOLIOSE AND FRUTICOSE THALLUS

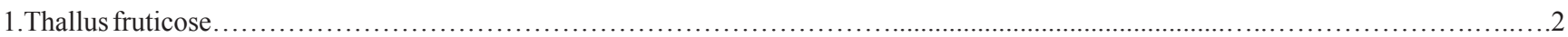

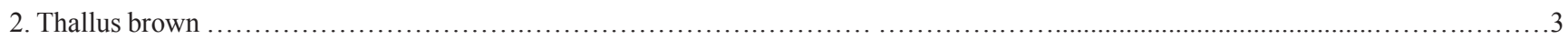

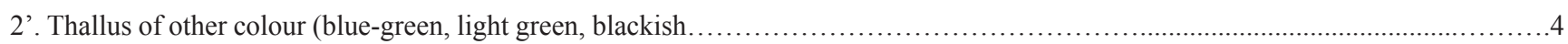

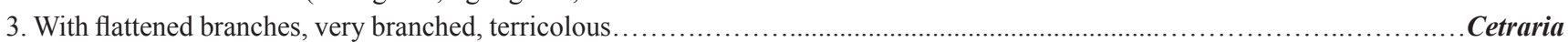

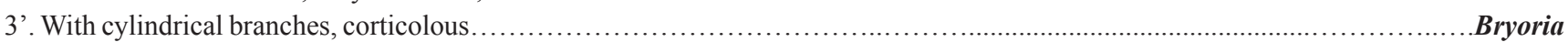

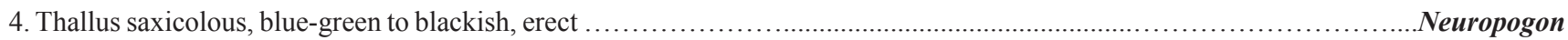

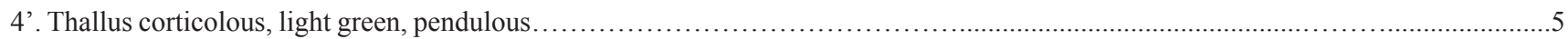

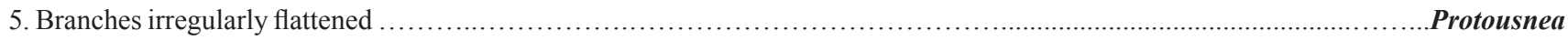

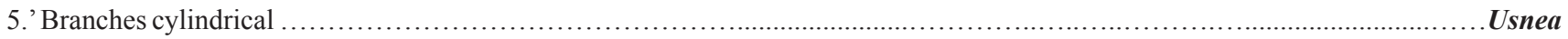

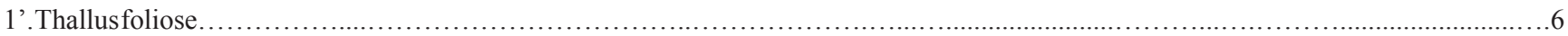

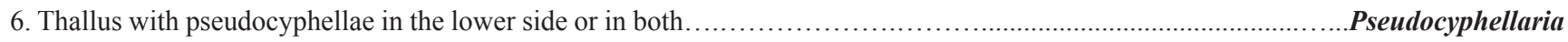

6'. Thallus without pseudocyphellae, but only occasionally near the ends of the lobules............................................................

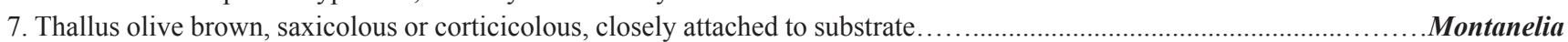

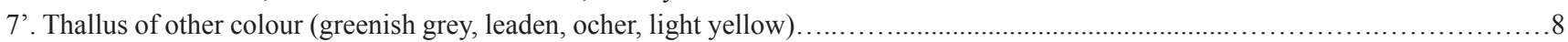

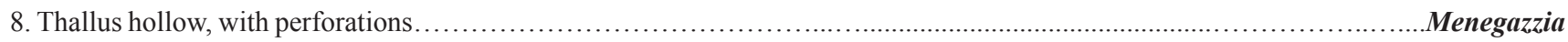

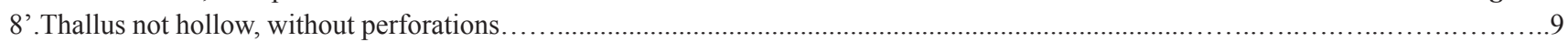

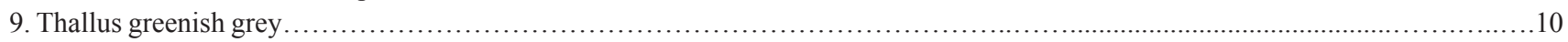

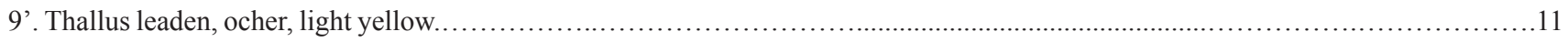

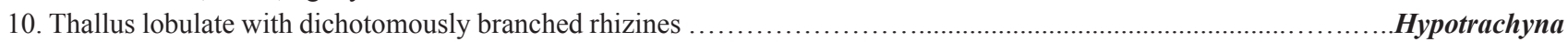

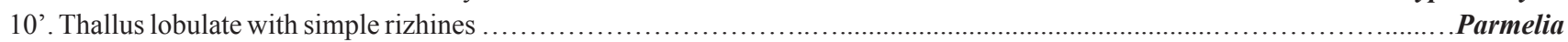

11. Thallus leaden, terricolous, marginal brown apothecia, photobiont Cyanobacteria ...............................................................Peltigera

11'. Thallus ocher to light yellow, saxicolous, laminal apothecia or absent, photobiont Chlorophyte...............................................12

12. Thallus ocher, apothecia common, laminal, with white disc, pruinose ........................................................................Rhizoplaca

12'. Thallus yellowish green. Apothecia rare, marginal, cinnamon-brown to dark brown, without pruina ..............Xanthoparmelia 
LiCHENS WITH PULVERULENT, UMBILICATE, GELATINOUS AND SQUAMULOSE THALLUS

1. Thallus corticolous or terricolous....

2. Thallus gelatinous, foliose, brown to black.

Leptogium

2 '. Thallus not gelatinous, pulverulent, whitish green...

1 '. Thallussaxicolous.

3. Thallus squamulose or placoide, light or dark green

....3

3'. Thallus umbilicate, brown, black or grey

Squamarina

Umbilicaria

\section{CONCLUSIONS}

There is a direct relationship between the richness of saxicolous species and the highest altitude.

The greatest lichen richness is concentrated in the tree and shrub strata.

It requires to makes more efforts in the material collection that to allow increasing the knowledge about of the distribution of the lichen species in the region with the purpose to establish the true status of conservation of the species in the region.

Also is necessary to increase the taxonomic studies for more of the genera of lichens in Chile used different taxonomic tools.

Lichenological expeditions should be continued in unexplored localities of Chile with emphasis on lichen biodiversity.

\section{ACKNOWLEDGEMENTS}

This work was funded by the Instituto de Ciencias Biológicas; Instituto de Química de Recursos Naturales, Universidad de Talca (Talca, Chile); PIEI-QUIBIO (UTalca); the National Research Foundation of Korea (No. 2011-0030491) and the Foundation of Key Laboratory, CAS (KLBB-201210).

\section{REFERENCES}

Arup, U., U. Sochting \& P. FrödÉn. 2013. A new taxonomy of the family Teloschistaceae, Nordic Journal of Botany 31(1): 16-83.

Arroyo, M.T.K., F. Squeo, L. Cavieres \& C. Marticorena. 2004. Chilenische Anden. In C.A. Burga, F. Klötzli \& G. Grabberr (eds.), Gebirge der Erde. Landschaft, Klima, Pflanzen Welt, pp. 210-219. Ulmer, Stuttgart, Germany.

Galloway, D.J. 1992a. Lichens of Laguna San Rafael, Parque Nacional 'Laguna San Rafael' southern Chile: indicators of environmental change. Global Ecology and Biogeography Letters 2: 37-45.

Galloway, D.J. 1992b. Studies in Pseudocyphellaria (lichens) III. The South American species. Bibliotheca Lichenologica 46: $1-275$.

Galloway, D.J. \& J.J. Elix. 2013. Reinstatement of Crocodia Link (Lobariaceae: Ascomycota) for five species formerly included in Pseudocyphellaria Vain. Australasian Lichenology 72: 32-42.

Kantvilas, G. \& P.W. James. 1987. The Macrolichens of Tasmanian rainforest: Key and notes. The Lichenologist. 19(1):1-28.

Orange, A., P.W. James \& F.J. White. 2010. Microchemical methods for the identification $\left(2^{\text {nd }}\right.$ Ed.). British Lichen Society, London. $101 \mathrm{pp}$.

Pereira, I. 2007. Mycobiota liquenizada del Parque Katalapi, X Región, Chile. Gayana Botanica 64: 192-199.

Pereira, I. \& J. SAn Martín. 1998. Flora liquénica corticícola en un bosque caducifolio de Nothofagus alesandrii de Chile Central. Cryptogamie, Bryolologie. Lichenologie 19(1): 59-72.

Pereira, I., J. San Martín \& C.L. Roux. 1999. Patrón florístico de Líquenes Epífitos de Nothofagus glauca en un bosque costero de Chile Central. Gayana Botanica 56(2): 69-76.

Pereira, I., J. San Martín \& M. Moya. 2002. Epiphytic Lichens on Gomortega keule (Gomortegaceae) coastal mountain (Central Chile). Mitteilungen aus Institut für Allgemeine Botanik, Hamburg 30-32: 171-185.

Quilhot, W., M. Cuellar, R. Díaz, F. Riquelme \& C. Rubio. 2012. Lichens of Aysén southern Chile. Gayana Botanica 69(1): 57-87.

Rizzi, G., G. Incerti, F. Ginaldi, D. Kodnik, S. Viglioni \& P. GIORDANI. 2011. A contribution to the flora of Sardinia. Mycotaxon 115: 535.

Roux, C. 2012. Liste des lichens et champignons lichénicoles de France. Listo de la likenoj kaj nelikeniĝintaj fungoj de Francio Bulletin de la Société Linnéenne, Provence, $n^{\circ}$ spécial 16: 1-220.

Rubio, C., M. Saavedra, M. Cuéllar, R. Díaz \& W. Quilhot. 2013. Epiphytic lichens of Conguillio National Park, southern Chile. Gayana Botanica 70(1): 66-81.

Santesson, R. 1942. The South American Menegazziae. Arkiv för Botanik 30A, (11): 1-35.

Stenroos, S. 1995. Cladoniaceae (Lecanorales, Lichenized, Ascomycotina) in the flora of Chile. Gayana Botanica 53: 89-131.

Vargas Castillo, R. \& C. Morano Büchner. 2014. Hongos liquenizados en morrenas del monte San Lorenzo, Región de Aisén, Chile. Gayana Botanica 71(1): 140-146.

Villagra, J., D. Montenegro, C. San Martín, C. Ramírez \& I. Álvarez. 2009. Estudio de la flora liquénica de las turberas de la comuna de Tortel (Región de Aisén) Patagonia Chilena. Anales Instituto Patagonia (Chile) 37(1): 53-62.

Vinayaka, K.S., S. Nayaka, Y.L. Krishnamurthy \& D.K. Upteri. 2012. A report on some macrolichens new to Karnataka, India. Journal of Threatened Taxa 4(1): 2318-2321.

ZAHLBRUCKNER, A. (1938-1940). Catalogus Lichenum Universalis 10: $1-660$.

Recibido: 19.08 .14

Aceptado: 22.10.15 